\title{
Effects of a group mindfulness-based cognitive programme on smartphone addictive symptoms and resilience among adolescents: study protocol of a cluster- randomized controlled trial
}

\author{
Anson Chui Yan Tang ${ }^{1 *}$ and Regina Lai Tong Lee ${ }^{2}$
}

\begin{abstract}
Background: Smartphone addiction in adolescent is a significant global health issue since the last decade. Evidence has shown that the uncontrolled use of smartphone would lead to undesirable impact on their growth and development. However, evidence-based interventions to manage adolescents' smartphone addictive behaviors is lacking. The proposed study aims to examine the effect of a group mindfulness-based cognitive programme(MBCP) on resilience, smartphone behavior and addictive symptoms in young adolescents.

Methods: It is an open-label, parallel-group, cluster-randomized controlled trial with repeated measurement analysis. Four primary schools in Hong Kong will be recruited and randomly allocated in a ratio of 1:1 to the intervention/control group. A convenience sample of 240 class level 5 primary school students, 60 from each school, will be recruited. Participants in the intervention group will receive a 12-week MBCP which comprises 90minute supervised practice at school and daily home practice. Resilience will be measured by Connor-Davidson Resilience Scale - 25 (Chinese version); smartphone behavior will be represented by time spent/day using the smartphone and types of functions used; addictive symptoms will be measured by Smartphone Addiction ScaleShort Version (Chinese Version). Baseline assessment(T0) will be conducted before the intervention starts. Post-tests will be conducted in weeks 4, 8, 12 of the intervention, and 3 months follow-up. Intention-to-Treat analysis will be applied to the variables. Generalized Estimating Equation model will be used to compare differences in resilience scores, smartphone behavior and addiction scores between and within groups, adjusted for socio-demographic factors. $P<0.05$ with two-tailed test will be regarded as significance.

Discussion: It is expected that adolescents will demonstrate better resilience and lesser smartphone addictive symptoms after joining the MBCP. The study will be the first provided empirical evidence to support the promising application of MBCP to manage smartphone use among adolescents. It introduces community stakeholders including community nurses a non-invasive and simple-to-administer intervention to tackle problematic smartphone use among adolescent clients.
\end{abstract}

\footnotetext{
* Correspondence: ansontang@twc.edu.hk

'School of Nursing, Tung Wah College, Hong Kong, China

Full list of author information is available at the end of the article
}

(C) The Author(s). 2021 Open Access This article is licensed under a Creative Commons Attribution 4.0 International License, which permits use, sharing, adaptation, distribution and reproduction in any medium or format, as long as you give appropriate credit to the original author(s) and the source, provide a link to the Creative Commons licence, and indicate if changes were made. The images or other third party material in this article are included in the article's Creative Commons licence, unless indicated otherwise in a credit line to the material. If material is not included in the article's Creative Commons licence and your intended use is not permitted by statutory regulation or exceeds the permitted use, you will need to obtain permission directly from the copyright holder. To view a copy of this licence, visit http://creativecommons.org/licenses/by/4.0/. The Creative Commons Public Domain Dedication waiver (http://creativecommons.org/publicdomain/zero/1.0/) applies to the data made available in this article, unless otherwise stated in a credit line to the data. 
Trial registration: Chinese Clinical Trial Registry, ChiCTR2000033273, Registered on 26 May 2020.

Keywords: smartphone addiction, smartphone behavior, adolescents, mindfulness, cognitive therapy, cluster randomized controlled trial, nursing

\section{Background}

The smartphone has become indispensable in the lives of most of humankind during the last two decades $[1,2]$. More than $75 \%$ of families own mobile devices such as smartphones [3]. Recent statistics from local and national surveys reveal a rapidly increasing trend in the use and possession of smartphones not only among adults but also among children and adolescents [4]. In 2011, $52 \%$ of children below 9 years of age had access to mobile devices; in 2013, that access rate had increased to $75 \%$ [5]. In 2018, about half of the elementary school students in the United State and Europe, possessed their own mobile phone [6]. In 2009, the average screen time of 8- to 18-year-old youngsters was $7.38 \mathrm{~h}$, which is $1.17 \mathrm{~h}$ more than the one reported in 1999 [7]. A similar pattern has been observed in Hong Kong. A survey about the use of smartphones among 10- to 24-year-old adolescents and young adults revealed that $88.8 \%$ of the respondents possessed a smartphone [8]. The older the age, the higher the possession rate [1]. The average time spent on screen media increased $15 \%$ in nine years' time, from 2003 to 2012. In particular, young adolescents aged 10 to 14 years showed the largest increase in screen time. Their average duration spent on screen media per week increased from $14.6 \mathrm{~h}$ to 2003 to $18.5 \mathrm{~h}$ in 2013 (an increase of nearly $27 \%$ ) [4].

The continuously increasing possession and use of mobile devices among children and adolescents is alarming because both their bodies and minds have not yet fully developed; in particular, their self-discipline is weak. The uncontrolled use of these mobile devices will make them even more susceptible than adults to the negative effects of mobile devices on their physiopsycho-social health [1]. Preliminary evidence shows that prolonged engagement with these devices disrupts cognitive development and leads to various physiological and psychosocial problems, including but not limited to visual problems, disruptions of memory, decline of attention, addiction, learning and social problems $[1,9$, 10]. Furthermore, breaking addictive patterns early in life will help them maintain the benefits into adulthood. The research team therefore focuses on investigating effective intervention to manage the addictive smartphone behavior of this vulnerable group.

Smartphone behavior includes the daily duration and frequency of smartphone use and types of functions used. The findings among studies about the pattern of using smartphone in adolescents vary with the countries of origin and target groups [9]. Social networking is one of the popular smartphone functions being used most by adolescents and adults [1115]. Some evidence shows that adolescents using smartphones for social networking are more likely to become smartphone addicts [11, 15]. Watching television content online with a smartphone is popular among younger children in Europe and the United States $[14,16]$. Other activities such as seeking information, completing homework and killing time are common reasons youngsters stay online $[1,8,16,17]$. Department of Health (2018) conducted a focus group interview to explore the views of using the internet and electronic screen products among school students in Hong Kong [1]. It showed that playing games was a major activity to relieve students' stress created by heavy homework. Van Deursen et al. (2015) reported that both process- and social-related gratifications were associated with addictive smartphone behaviors in adults [15].

Uncontrollable use of smartphone is problematic and will lead to addiction to the smartphone use in the worst cases. Smartphone addiction is being perceived as a unique type of behavioral addiction distinguished from internet addiction [18]. The smartphone is a portable electronic device which has the functions of a telephone as well as the capabilities of a computer. It can be used for communication; for internetbased activities; and for personal purposes through the many "apps" available $[11,19]$. Symptoms of smartphone addiction are (1) smartphone is his companion, on which he/she relies to can produce pleasure and relieve stress; (2) a person is unable to control his use of the smartphone, particularly for engaging with social media, etc.; and (3) the uncontrolled use has negative effects on his/her financial, physical, psychological and social aspects $[20,21]$. Park et al. (2018) reported that about $11 \%$ of South Korean adolescents were at high risk of smartphone addiction which required further assessment and intervention [22]. Haug et al. (2015) found that $17 \%$ of adolescents and young adults in Switzerland were addicted to smartphone [11]. In Hong Kong, 19-27\% of 11- to 18-year-old adolescents were found to be either at risk of internet addiction or were confirmed internet addicts (Shek et al., 2008; Shek \& Yu, 2012) 
[23, 24]. Some studies showed that gender, age, family characteristics are significantly associated with smartphone addiction [15, 22, 25].

A growing body of evidence demonstrated that such smartphone addiction is associated with numerous problems in physical, psychological and social health of children and adolescents. Excessive smartphone use is found to be associated with poor sleep quality, cardiovascular risk factors, attention-deficit hyperactivity, and depression and anxiety symptoms [26-28]. It is also shown that smartphone addiction is associated with behavioral and sexual issues commonly identified in adolescents such as antisocial behaviors, smoking, alcohol consumption, more sexually active $[27,29,30]$. The significant linkage of smartphone addiction with a wide range of physiopsychosocial problems among adolescents urges nurses especially those working school and other youth health care settings to look for effective preventive measures to tackle the uncontrollable smartphone use among adolescents prior to the occurrences of negative heath consequences.

\section{Resilience as a potential protective factor for smartphone addiction}

Previous observational studies showed that psychological traits such as resilience may better predict smartphone addition than the pattern of smartphone behavior [31, 32]. Resilience is one's ability to maintain psychological wellbeing and adapt to stress or difficult situations successfully [33, 34]. Factors affecting resilience development include intrinsic characteristics of an individual such as strong emotion regulation, high coping selfefficacy; child's relationships with parents and social factors such as quality of schools and neighborhoods [35]. Low resilience has been found to be associated with internet/smartphone addiction. Strong resilience was found to be a key factor in protecting adolescents from experiencing online risks. It can also neutralize the negative psychological effects associated with internet addiction and online risk exposure [36]. 9-10 year-old youngsters with low resilience had a higher risk of becoming internet addicts [37-39]. Kim et al. (2014) examined the association of depression, impulsiveness and resilience on smartphone addiction in university students in South Korea. It reported that the addiction group spent more time using smartphones on weekdays than the non-addiction group. The addiction group had a significantly lower resilience score measured by Conner-Davidson Resilience Scale than the nonaddiction group [27]. Jung and Kim (2015) found that adolescents with high addiction risk had a significantly lower ego resilience than those with potential or no risk [40]. More recent studies showed that resilience have a mediating effect on smartphone dependency and depression and aggressiveness among adolescents though the mediating effects may be different between sexes in internet addiction [32, 41-43]. The current evidence suggests that resilience can play a role in modulating addictive behavior of smartphone users. Increasing one's resilience may help to prevent the addiction. It implicates nurses to look for evidence-based intervention to strengthen resilience to minimize health issues associated with smartphone addiction.

\section{Mindfulness-Based Cognitive Therapy (MBCT)}

Little research has been conducted so far regarding effective management of smartphone addiction [44]. It is proposed that a person's subconscious addictive behavior may be corrected by $[45,46]$. Mindfulness is a kind of meditation originating from Buddhist practice. It aims to engage an individual in a full, direct, active and continuous awareness of experienced phenomena [46]. Available evidence supports that practicing mindfulness during childhood and adolescence may facilitate healthy psychosocial development such as strengthening executive function, increasing affective self-regulation, increasing the ability to moderate strong emotional states, and improving interpersonal relationships [47-50]. Studies investigating the role of mindfulness in managing behavioral addiction have primarily focused on problem gambling and work addiction, and they have rarely targeted children and/or adolescents [45]. The effect of mindfulness on smartphone addiction is yet to be explored [51]. Nonetheless, strong emotion regulation is one of prominent psychosocial characteristics of resilience [35]. Enhancing adolescents' emotion regulation through mindfulness-related activities may possibly help to increase their resilience and so to modulate the smartphone behavior. Lee et al. (2008) developed a mindfulness-based cognitive therapy for 8- to 12-yearold children (MBCT-C) based on the adult MBCT developed by Segal et al. (2002) [52, 53]. It was intended to increase social-emotional resilience through strengthening mindful attention of children with anxiety problems [54]. A pilot study conducted by Lee et al. (2008) showed that the MBCT-C was feasible with children and that it could relieve their attention and anxiety problems [52]. Semple et al. (2010) conducted a randomized controlled trial to evaluate MBCT-C on 25 children aged 9 to 12 years [54]. Consistent trends of reduced anxiety and depressive symptoms were observed for the participants completing a full course of the therapy. In addition, $61 \%$ of the parents reported that fewer conduct or anger management problems were observed on their children after participating in the programme. Although the small sample size may compromise the validity of these findings, it suggests the potential of MCBT$\mathrm{C}$ to manage children with emotional and behavioral 
problems. As such, the research team will apply this therapy with adolescents and evaluate its effect on their resilience, smartphone behavior and smartphone addictive symptoms. This research protocol presents the design of this study and discuss the potential nursing implication of the study findings.

\section{Methods/Design}

\section{Aim/Objectives}

The aim of this study is to examine, through a cluster randomized controlled trial, the effects of a group mindfulness-based cognitive programme on smartphone behavior, resilience and symptoms of smartphone addiction in adolescents.

The primary objective is to investigate the group by the time interaction effects of the group mindfulnessbased cognitive programme on resilience, smartphone behavior and the symptoms of smartphone addiction in adolescents.

There are two secondary objectives: (1) To identify adolescents with smartphone addictive symptoms using the Smartphone Addiction Scale-Short Version; (2) To investigate the association of socio-demographic factors, smartphone behavior and resilience with smartphone addiction symptoms in adolescents.

\section{Hypotheses}

The three primary hypotheses of this study are: (1) the smartphone behavior of adolescents can be improved by participating in a group mindfulness-based cognitive programme; (2) the smartphone addictive symptoms of adolescents can be reduced by participating in a group mindfulness-based cognitive programme; (3) the resilience of adolescents can be increased by participating in a group mindfulness-based cognitive programme. The secondary hypothesis is that socio-demographic characteristics, resilience and smartphone behavior are associated with the symptoms of smartphone addiction in adolescents.

\section{Study design and setting}

This study will be an open-label, parallel-group, cluster randomized controlled trial with repeated measurement analysis. It will pragmatically evaluate the effects of the mindfulness-based cognitive programme (MBCP) on improving adolescents' resilience, changing their smartphone behavior and reducing smartphone addictive symptoms as compared with the control group at five measurement points (i.e. baseline (T0), one month (T1), two months (T2), three months (T3) after the programme, three months' follow up (T4)). The study will be conducted in four primary schools in Hong Kong. The primary schools will be the unit of allocation, and individual participants will be the units of analysis.
The intervention group will receive the mindfulnessbased programme, and the control group will receive existing counselling services for students with emotional/behavioral problems. (i.e. treatment as usual). Data collection will be started in September 2020. Key aspects of the study design can be found in Fig. 1. We designed and reported this study protocol according to the SPIRIT2013 statement.

\section{School and participant recruitment}

Primary school will be the cluster for randomization. Four primary schools in Hong Kong will be recruited based on their eligibility and willingness to participate in the study. The inclusion criteria for school selection are: (1) it is either a local government-subsidized or a private primary school; and (2) there are currently no interventions adopted by the school to manage students' smartphone behavior. The exclusion criteria are (1) it is an international school; and (2) there are currently interventions being implemented by the school to manage students' smartphone behavior.

A convenience sample of 240 students will then be recruited from the selected schools (i.e., 60 students from each school) based on their eligibility and consent to participate. The inclusion criteria are: (1) adolescents are aged 10-11 years; (2) adolescents are enrolling in class level 5; (3) adolescents possess a smartphone or can access a smartphone; (4) adolescents can speak, understand and write Cantonese; (5) adolescents are cognitively capable of following simple instructions. Adolescents have intellectual/learning disabilities will be excluded from the selection.

\section{Sample size calculation}

As it is the first study investigating the effect of the MBCP on smartphone behavior in adolescents, the project team can only decide the predicted intracluster correlation with reference to other studies investigating adolescent behavioral problems. The predicted intracluster correlation of 0.01 is adopted based on studies about smoking prevention and alcohol use in adolescents [55, 56]. $20 \%$ potential dropout/noncompliance rate is considered reasonable. Assuming a Type 1 error of 0.05 and Type II error of 0.2 , a total of 240 participants (i.e. 120 in each group, 60 in each school) is required to achieve a medium effect (Cohen's $d$ ) of 0.5 [57] for comparing the treatment effect between the two treatment groups and among the measurement points within groups for the three outcomes to be measured - resilience, smartphone behavior and symptoms of smartphone addiction.

\section{Randomization and blinding}

The four participating schools will be clusterrandomized at a ratio of 1:1 into the intervention and 


\begin{tabular}{|c|c|c|c|c|c|c|c|c|}
\hline \multirow[b]{3}{*}{$\begin{array}{r}\text { TIMEPOINT } \\
\text { (Week) } \\
\end{array}$} & \multicolumn{8}{|c|}{ STUDY PERIOD } \\
\hline & \multirow{2}{*}{$\begin{array}{c}\text { Enrolment } \\
-20 \sim-1 \\
\end{array}$} & \multirow{2}{*}{$\frac{\text { Allocation }}{0}$} & \multicolumn{5}{|c|}{ Post-allocation } & \multirow{2}{*}{$\frac{\text { Close-out }}{36}$} \\
\hline & & & $1(\mathrm{TO})$ & $4(T 1)$ & $8(T 2)$ & $12(T 3)$ & $24(T 4)$ & \\
\hline \multicolumn{9}{|l|}{ ENROLMENT: } \\
\hline $\begin{array}{r}\text { School eligibility } \\
\text { screen }\end{array}$ & $\mathrm{X}$ & & & & & & & \\
\hline School permission & $\mathrm{X}$ & & & & & & & \\
\hline \multirow{2}{*}{$\begin{array}{r}\text { School randomization } \\
\text { and allocation } \\
\text { Parental informed } \\
\text { consent }\end{array}$} & & $\mathrm{X}$ & & & & & & \\
\hline & & $\mathrm{X}$ & & & & & & \\
\hline \multicolumn{9}{|l|}{ INTERVENTIONS: } \\
\hline \multicolumn{9}{|l|}{$\begin{array}{r}\text { Mindfulness-based } \\
\text { cognitive programme }\end{array}$} \\
\hline \multicolumn{9}{|l|}{ Control } \\
\hline \multicolumn{9}{|l|}{ ASSESSMENTS: } \\
\hline \multirow{2}{*}{$\begin{array}{r}\text { Socio-demographic } \\
\text { variables } \\
\text { Smartphone behavior }\end{array}$} & & & $\mathrm{X}$ & $\mathrm{X}$ & $\mathrm{X}$ & $\mathrm{X}$ & $\mathrm{X}$ & \\
\hline & & & $\mathrm{X}$ & $\mathrm{X}$ & $\mathrm{X}$ & $\mathrm{X}$ & $\mathrm{X}$ & \\
\hline \multirow{2}{*}{$\begin{array}{r}\text { Resilience } \\
\text { Smartphone addictive } \\
\text { symptoms }\end{array}$} & & & $\mathrm{X}$ & $\mathrm{X}$ & $\mathrm{X}$ & $\mathrm{X}$ & $\mathrm{X}$ & \\
\hline & & & $\mathrm{X}$ & $\mathrm{X}$ & $\mathrm{X}$ & $\mathrm{X}$ & $\mathrm{X}$ & \\
\hline \multicolumn{9}{|l|}{$\begin{array}{l}\text { PROCESS } \\
\text { EVALUATION: }\end{array}$} \\
\hline \multicolumn{9}{|l|}{ Fidelity monitoring } \\
\hline \multicolumn{9}{|l|}{ DISSEMINATION: } \\
\hline Data analysis & & & & & & & & $\mathrm{X}$ \\
\hline Writing report & & & & & & & & $\mathrm{X}$ \\
\hline
\end{tabular}

Fig. 1 Standard Protocol Items: Recommendations for Interventional Trials (SPIRIT) Figure

control groups by coin toss by the research assistant A (RA-A) who will not be involved in participant recruitment and data collection. All students recruited under the same participating school were exposed to the same intervention. The principal investigator, who will be responsible for recruiting the schools and the students, will code the participating schools with a number and a letter to represent the district and the school, respectively. RA-A will only know the four codes but not the names of the schools. The school will be assigned to the intervention group if the landed side is heads; the school will be assigned to the control group if the landed side is tails. The allocation result will be kept by the RA-A in a sealed opaque envelope until participant recruitment. The school teachers, parents and students will not be informed of the research hypotheses. RA-A will feed data into the computer in separate datasheets so that the coinvestigator responsible for data analysis can analyze data without having access to information about the allocation. Hence, it is a single-blind design.

\section{Outcome measures \\ Demographics}

Demographic variables will be gender, age, parents' educational attainment, parents' employment status, family structure, parenting skills. Age will be measured in continuous scale; the other variables will be measured in nominal/ordinal scales.

\section{Smartphone behavior}

Smartphone behavior will be operationalized in terms of duration of daily smartphone use and types of smartphone functions used. Duration of daily smartphone use will be assessed by one closed-ended question - 'On average, how long do you use your smartphone on a typical day?'. The respondents will respond to the question 
by choosing among the six categories - 'less than $10 \mathrm{mi}$ nutes', '11-60 minutes', '1-2 hours', '3-4 hours', '5-6 hours', or 'more than 6 hours'. For types of smartphone functions used, the respondents will indicate the frequency of using a specific smartphone function such as social networking, phone calls, gaming, etc. in a 5-point Likert scale $(0=$ never, $1=$ rarely, $2=$ sometimes, $3=$ usually, 4 = always).

\section{Resilience}

Resilience will be measured by the Chinese Version of the Connor-Davidson Resilience Scale - 25 [58, 59]. It consists of 25 items reflecting five factors of resilience: (1) tenacity, (2) control, (3) spiritual influence, (4) positive acceptance of changes and secure relationship, and (5) tolerance of negative affect and strengthening effects of stress [58]. Each item consists of statements such as 'able to adapt to change', 'close and secure relationships', 'sometimes fate or God can help', 'can deal with whatever comes', etc. The respondents will be asked to rate their agreement with each statement based on their experiences in the previous month. If a particular situation has not arisen in that time, the respondents will be instructed to respond according to how they think they would have reacted. The respondents will be directed to indicate their response on each item with a 5-point Likert scale, 0 is 'not true at all' and 4 is 'true all the time'. The total resilience score ranges from 0 to 100 , with higher scores indicating greater resilience. The scale has been applied to various target populations including children 10 years old and above. A Flesch-Kincaid score of 5 indicates that the scale is expected to be understood by those with a fifth-grade level education that is 10 years of age [60]. Thus, the scale is suitable for the target group in this study. Regarding the validity and reliability of the Chinese version, the Cronbach's alpha coefficient was 0.89 . The resilience score had positive correlation with social support $(r=0.44)$ and negative correlations with depression $(\mathrm{r}=-0.38)$ and anxiety $(\mathrm{r}=-0.25)$ [59]. To ensure the scale is appropriate and comprehensible to class level 5 Hong Kong primary school students, a panel of primary school teachers will be invited to review the scale and make minor modifications in the wordings. Face validity, internal consistency and test-retest reliability will be conducted with 20 adolescents before the actual data collection.

\section{Smartphone addictive symptoms}

Smartphone additive symptoms will be assessed using the Smartphone Addiction Scale-Short Version. This 10item self-administered questionnaire was developed by Kwon et al., 2013 based on the original validated Smartphone Addiction Scale [18]. The scale addresses five domains of smartphone addiction: (1) daily-life disturbance, (2) withdrawal, (3) cyberspace-oriented relationship, (4) overuse and (5) tolerance. The respondents will rate their agreement with each item on a 6-point Likert scale, from 1 representing 'strongly disagree' to 6 , representing 'strongly agree'. The total addiction score possible ranges from 10 to 60 . The higher the score, the more severe the addictive symptoms. The internal consistency (Cronbach's alpha) was 0.911. Analysis of receiver operating characteristics demonstrated that for boys, the sensitivity was 0.867 and the specificity was 0.893 . For girls, the sensitivity and the specificity were 0.875 and 0.886 respectively [18]. A Chinese-version of the scale will be translated based on the English version by backward translation, i.e. a native Chinese and English-speaking teacher. Face validity, internal consistency and test-retest reliability will be conducted by inviting 20 adolescents to fill out the questionnaires before actual data collection, to ensure the translated version is valid and reliable for this study.

\section{Intervention}

The participants allocated to the intervention group will receive a 12-week group mindfulness-based cognitive programme (MBCP) adapted from the MCBT-C developed by Lee et al. (2008) [52]. It consists of two components: weekly 90-minute supervised practice, and daily 15 min home practice. Supervised practice will be conducted in a group of 20 at a quiet room of the primary school. In the first supervised practice session, the trainer will inform the participants the rules of the practice sessions, for example, 'we can remember not to talk when another person is talking.', 'we agree not to talk during mindful awareness practices so as not to disturb others.', etc.

Participants will be directed to perform a short breath meditation at the beginning and at the end of each session. To engage the young participants in the session, the trainer, who has years of experience in providing mindfulness and cognitive therapy to children, will use a variety of short sensory-based movement activities that are practiced repeatedly in 3- to 5-minute blocks. These simple sensory exercises can enhance their mindful awareness by helping them to experience internal and external environments non-judgmentally through various senses, i.e. sight, sound, touch, taste, smell and kinesthetic. Examples of these activities are sensorybased practices, seated breath meditations, mindful movement activities, body scans, visualization practices, and drawing and writing [51]. If there are participants with food allergies, the trainer will not select the allergic foods for the eating/smelling/touching activities. The parents will be informed the food that will be used for training in the week before each session in writing. 
Aside from the supervised practice, the participants will be introduced to three to four home-practice activities during each supervised session. They will be encouraged to perform these activities for about $15 \mathrm{~min}$ a day, six days a week. Each participant will have his/her own logbook labeled with his/her code to record the home practices. The trainer will review the practice record every week. Those who have not performed the home-practice activities will be reminded by the trainer to do them in the coming week to increase the compliance. Details of the mindfulness-based cognitive programme can be found in Table 1.

Over the data collection period, the controls will not exposed to any interventions except counselling services that normally provided to students in need by social workers at the school due to emotional/behavioral issues.

\section{Safety monitoring}

During the training session, another research assistant (RA-B), who is a qualified registered nurse, will closely monitor whether the participating students have allergic symptoms such as itchiness, skin rash, etc. If a student has these symptoms, the trainer will inform the responsible teacher immediately. The parents will also be contacted to explain the allergic reaction and advise them to consult their family doctor for detailed assessment. In case the student manifests severe allergic reactions, the trainer and the research assistant will follow the guidelines and emergency care plan on food allergy and anaphylaxis of the schools to call emergency service and provide basic life support to the student immediately.

\section{Data collection}

The information sheets, the consent forms, the questionnaires and envelopes will be distributed to the parents of the class level 5 students in the participating primary schools via class teachers. The parents will be required to indicate the food(s) their child is allergic to, if any, on the consent form. The parents who are willing to allow their children to participate in the study will return signed consent forms and completed questionnaires in sealed envelopes to the school before starting the intervention (T0). The trainer will record the attendance of each participant in every session throughout the 12week intervention. To follow up on the effect of the intervention on the participants, post-intervention assessments will be conducted on all outcomes in week 4, week 8 , by the end (week 12) of the programme and 3 months follow-up (T1-T4). It will take about $20 \mathrm{~min}$ to complete the questionnaire. RA-B will be responsible for collecting all the questionnaires. The school teachers will not be involved in the data collection. Figure 2 shows the trial flow.

\section{Validity and reliability/rigour}

To ensure intervention fidelity and adherence, structured observations of a randomly selected session per week will be performed by RA-B. The project team will first develop quality scales to assess the fidelity quantitatively. The quality scales will be session-specific. For the home practice, each participant will have a logbook to record their compliance to the recommended homepractice activities. In addition to the reminder to the participants regarding the home practices by the end of every supervised session, a circular will also be sent to their parents to solicit their help to monitor their children for the home practices every week to increase the treatment adherence.

As both participants in the intervention and control groups are allowed to receive counselling services provided by the school social workers if necessary to handle some emotional/behavioral issues, a record of whom have received the service and how often will be kept to facilitate an interpretation of the study findings.

\section{Data analysis}

IBM SPSS version 23 will be used for statistical analysis. Mean and standard deviation will be reported for continuous variables, i.e. age, types of smartphone functions used and total resilience score. Frequency and percentage will be reported for nominal and ordinal variables. Cronbach's alpha and intra-class correlation coefficients will be calculated to determine the validities and reliabilities of the two scales: the translated Smartphone Addiction Scale and the Chinese Version of Conner-Davidson Resilience Scale - 25. Independent t-test/Mann-Whitney $\mathrm{U}$ test will be used to compare the socio-demographic variables between intervention and control groups. Generalized Estimating Equation model will be used to verify all the hypotheses. The socio-demographic variables will be treated as co-variates to adjust the group means. Results will be reported with $95 \%$ confidence interval. Intention-to-treat analyses will be applied to the independent and dependent variables. Missing data will be handled by replacing the missing values with the itemspecific mean. Tests performed will be two-tailed with alpha value set at 0.05 . Cohen's $d$, calculated by taking the difference of the adjusted means between two comparison groups and dividing it by the pooled standard deviation, will be used to estimate the effect size of the treatment.

\section{Discussion}

The expected outcome of this study is that the mindfulness-based cognitive programme will lead to improvements in resilience and reduction of smartphone addiction risk among adolescents. The MBCP enables the adolescents to grasp a new skill to manage their 


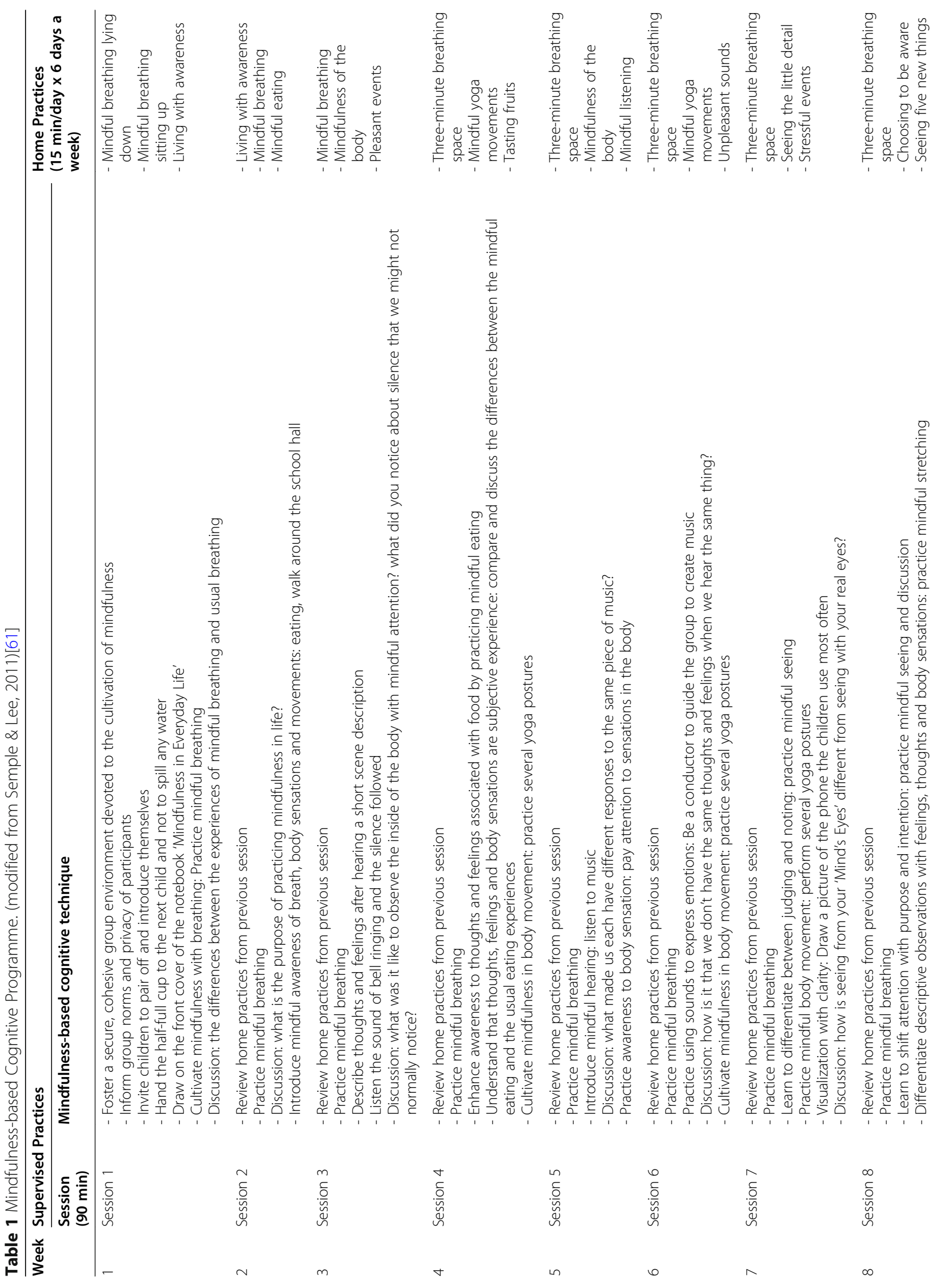




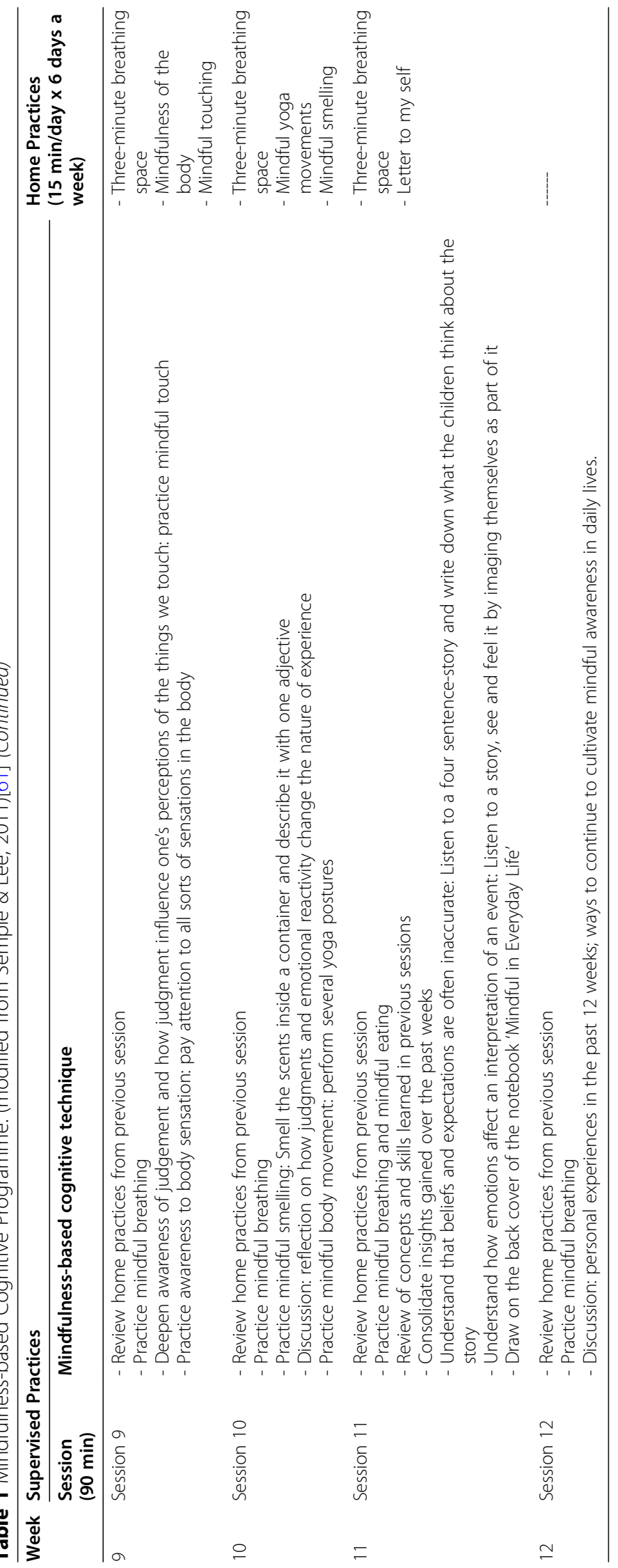


School recruitment:

- Inclusion criteria: either a local government-subsidized or a private primary school; currently no interventions at school to manage students' smartphone behaviors.

- Exclusion criteria: international school and currently has interventions at school to manage students' smartphone behaviors.

\section{Enrolment}

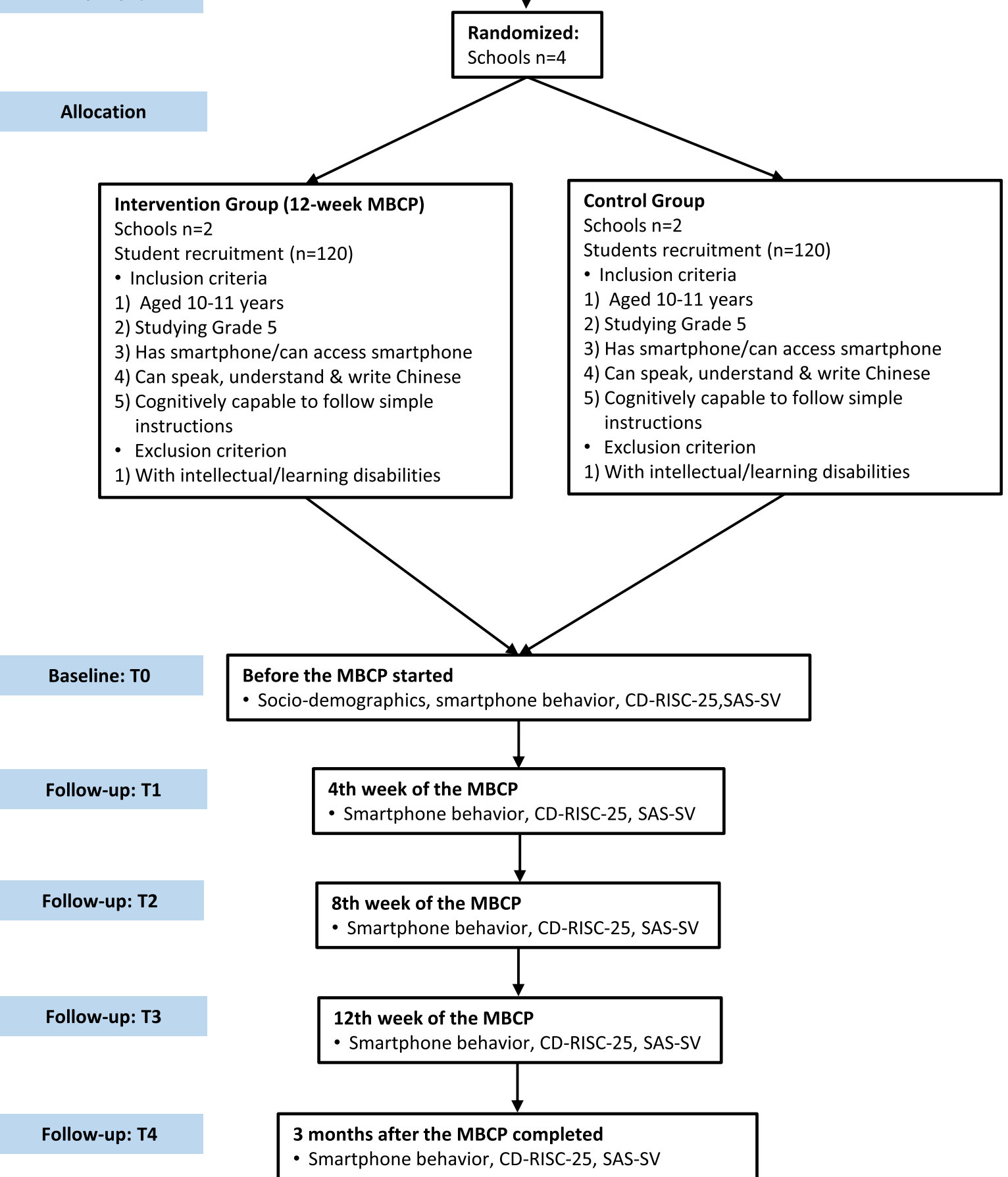

Fig. 2 Trial Flow Chart 
emotion by attending to the present and modify their cognitive thoughts toward stress and difficulties encountered in life. Throughout the training process, the adolescents will learn that they can manage their emotion through daily mindfulness practice. The programme has a lifelong benefit to adolescents as it enables them to manage their stress and conflicting thoughts which linger in adolescents with a simple and economical approach.

Problematic smartphone use, when not managed timely and adequately, may progress to smartphone addiction and other associated negative health outcomes. Our intervention can be served as a preventive intervention to be applied regularly in schools and other adolescent health centers to strengthen adolescents' resilience in order to boost up their resistance against addictive smartphone use. Nurses, being one of the major frontline healthcare providers in the community to monitor people's health and provide health promoting intervention, could integrate this noninvasive and simple-toadminister intervention into existing adolescent wellness programmes to not merely manage smartphone use issue but also to promote the overall wellbeing of the adolescent clients.

It is hoped that findings from the study could lead to larger, multisite trials to provide more robust evidence. In addition, similar evidence-based approaches can be developed in the future for other age groups who are vulnerable to smartphone addiction to strengthen the protection against the negative effects of smartphone use.

There are several limitations of this protocol. This study targets early adolescents; the generalizability of the study findings to middle- and late-adolescents is unknown. As two treatment groups will receive treatments in totally different formats, ascertainment bias might be introduced to the results although the study hypotheses will not be made known to the schools, the parents and the students. Only four primary schools will be recruited; the similarity in characteristics exhibited by participants from the same school may bias the results. To assess the impact of potential clustering of the participants from the same school, Generalized Estimating Equation model will be adopted to control the cluster effect of school.

\section{Abbreviations}

MBCT: Mindfulness-based cognitive therapy.; MBCT-C: Mindfulness-based cognitive therapy for children.; MBCP: Mindfulness-based cognitive programme.; RA-A: the first research assistant.; RA-B: the second research assistant.; CD-RISC-25: Connor-Davidson Resilience Scale - 25.; SASSV: Smartphone Addiction Scale-Short Version.

\section{Acknowledgements}

I would like to express my gratitude to Ms. Liz Tong, who have been devoted to developing and delivering the intervention protocol.

\section{Authors' contributions}

RL and AT conceptualized and design the research plan. RL is responsible for recruiting schools and data analysis. AT is responsible for data collection and intervention development. AT was a major contributor in writing manuscript. All authors read and approved the final manuscript.

\section{Funding}

This project is supported by Tung Wah College (Grant number: CRG2018/02). Research funders did not participate in research design, data collection, data analysis and publishing writing.

\section{Availability of data and materials \\ Not applicable.}

\section{Declarations}

Ethics approval and consent to participate

Ethics approval has been obtained from the Research Ethics Committee of Tung Wah College (reference number: REC2019046). Permission will be obtained from the participating primary schools before data collection. Information sheets, parents' written consents will be obtained from parents of the participants prior the intervention started.

\section{Consent for publication}

Not applicable.

\section{Competing interest}

The authors declare that they have no competing interests.

\section{Author details}

${ }^{1}$ School of Nursing, Tung Wah College, Hong Kong, China. ${ }^{2}$ School of Nursing and Midwifery, Faculty of Health and Medicine, The University of Newcastle, 2308 Callaghan, New South Wales, Australia.

Received: 7 May 2021 Accepted: 25 May 2021

Published online: 05 June 2021

\section{References}

1. Department of Health. E-Report: Report of advisory group on health effects of use of Internet and electronic screen products. The Hong Kong Special Administrative Region. 2018. https://www.studenthealth.gov.hk/english/ internet/report/files/e_report.pdf. Accessed Jun 2020.

2. Hardell $L$. Effects of mobile phones on children's and adolescent's health: A commentary. Child Dev. 2018;89(1):137-40. DOl:https://doi.org/10.1111/ cdev.12831.

3. Lauricella AR, Wartella E, Rideout VJ. Young children's screen time: The complex role of parent and child factors. Journal of Applied Developmental Psychology. 2015;36:11-7. DOl:https://doi.org/10.1016/j.appdev.2014.12.001.

4. Census and Statistics Department. Thematic Household Survey Report No. 43-52 Information Technology Usage and Internet Penetration. Hong Kong Special Administration Region; 2013.

5. Chassiakos YR, Radesky J, Christakis D, Moreno MA, Cross C. Children and adolescents and digital media. Pediatrics. 2016;138(5):e201625936. DOl: https://doi.org/10.1542/peds.2016-2593.

6. Odgers CL. Smartphones are bad for some adolescents, not all. Nature. 2018;554(7693):432-4. DOl:https://doi.org/10.1038/d41586-018-02109-8.

7. Magee CA, Lee JK, Vella SA. Bidirectional relationship between sleep duration and screen time in early childhood. JAMA Pediatrics. 2014;168(5): 465-70. DOI:

8. The Hong Kong Federation of Youth Groups. Smartphones and me Survey. The Hong Kong Federation of Youth Groups; 2013.

9. Durusoy R, Hassoy H, Ozkurt A, Karababa AO. Mobile phone use, school electromagnetic field levels and related symptoms: A cross-sectional survey among 2150 high school students in Izmir. Environ Health. 2017:16(51). https://doi.org/10.1186/s12940-017-0257-X.

10. Sadetzki S, Langer CE, Bruchim R, Kundi M, Merletti F, Vermeulen $R$, et al. The MODI_Kids study protocol: Challenges in assessing childhood and adolescent exposure to electromagnetic fields from wireless telecommunication technologies and possible association with brain tumor risk. Frontiers in Public Health. 2014;2(124). https://doi.org/10.3389/fpubh.2 014.00124. 
11. Haug S, Castro RZ, Kown M, Filler A, Kowatsch T, Schaub P. Smartphone and smartphone addiction among young people in Switzerland. Journal of Behavioral Addictions. 2015;4(4):299-307. DOl:https://doi.org/10.1556/2 006.4.2015.037.

12. Li S, Chung T. Internet function and internet addictive behavior. Comput Hum Behav. 2006:22(6):1067-71.

13. Lopez-Fernandez O, Honrubia-Serrano L, Freixa-Blanxart M, Gibson W. Prevalence of problematic mobile phone use in British adolescents. Cyberpsychology Behavior Social Networking. 2014;17:91-8.

14. Office of Communication. Children and parents: Media use and attitude report. United Kingdom; 2013.

15. Van Deursen AJAM, Bolle CL, Hegner SM, Kommers PAM. Modeling habitual and addictive smartphone behavior: The role of smartphone usage types, emotional intelligence, social stress, self-regulation, age and gender. Comput Hum Behav. 2015;45:411-20.

16. Holloway D, Green L, Livingstone S. Zero to eight. young children and their Internet use. London School of Economics and Politics, London: EU Kids Online. 2013. http://eprints.Ise.ac.uk/52630/1/Zero_to_eight.pdf Accessed on Jun 2020.

17. Wong YC. Research study on Internet education: Final report. YMCA of Hong Kong. Department of Social Work and Social Administration, The University of Hong Kong, Policy 21 Limited.; 2010.

18. Kwon M, Kim DJ, Cho H, Yang S. The smartphone addiction scale: Development and validation of a short version for adolescents. PLoS ONE. 2013;8(12):e83558. DOl:https://doi.org/10.1371/journal.pone.0083558.

19. Nielsen P, Fjuk $A$. The reality beyond the hype: Mobile internet is primarily an extension of pc-based internet. The Information Society. 2010;26(5):375-82.

20. Andreassen CS, Pallesen S. Social network site addiction - An overview. Curr Pharm Des. 2014;20:4053-61.

21. Young KS. Internet addiction: Symptoms, evaluation and treatment. Innovations in Clinical Practice. 1999;17:19-31.

22. Park H, Cho E. Smartphone addiction and depression: The mediating effects of self-esteem and resilience among middle school students. Journal of Korean Academy of Community Health Nursing. 2018;28(3):280-90. DOl: https://doi.org/10.12799/jkachn.2017.28.3.280.

23. Shek DTL, Tang VM, Lo CY. Internet addiction in Chinese adolescents in Hong Kong: assessment, profiles, and psychosocial correlates. Sci World J. 2008:8:766-87

24. Shek DTL, Yu L. Internet addiction phenomenon in early adolescents in Hong Kong. Sci World J. 2012;2012:104304. https://doi.org/10.1100/2012/1 04304.

25. Mok JY, Choi SW, Kim DJ, Choi JS, Lee J, Ahn H, et al. Latent class analysis on internet and smartphone addiction in college students. Neuropsychiatric Disorder Treatment. 2014;10:817-28. DOl:https://doi.org/10.2147/NDT.S59293.

26. Lissak G. Adverse physiological and psychological effects of screen time on children and adoelscents: Literature and case study. Environ Res. 2018;164: 149-57. DOl:https://doi.org/10.1016/j.envres.2018.01.015.

27. Kim SM, Huh HJ, Cho H, Kwon M, Choi JH, Ahn HJ, et al. The effect of depression, impulsivity, and resilience on smartphone addiction in university students. Journal of Korean Neuropsychiatric Association. 2014;53(4):214-20. DOl:https://doi.org/10.4306/jknpa.2014.53.4.214.

28. Wang PY, Chen KL, Yang SY, Lin PH. Relationship of sleep quality, smartphone dependence, and health-related behaviors in female junior college students. PLOS ONE. 2019;14(4):e0214769-9. DOl:https://doi.org/1 0.1371/journal.pone.0214769.

29. Choi SW, Kim DJ, Choi JS, Ahn H, Choi EJ, Song WY, et al. Comparison of risk and protective factors associated with smartphone addiction and Internet addiction. Journal of Behavioral Addictions. 2015;4(4):308-14. DOl: https://doi.org/10.1556/2006.4.2015.043.

30. Grant JE, Lust K, Chamberlain SR. Problematic smartphone use associated with greater alcohol consumption, mental health issues, poorer academic performance, and impulsivity. Journal of Behavioral Addictions. 2019;8(2): 335-42. DOl:https://doi.org/10.1556/2006.8.2019.32.

31. Jiang Q, Li Y. Factors affecting smartphone dependency among the young in China. Asian Journal of Communication. 2018;28(5):508-25. DOl:https:// doi.org/10.1080/01292986.2018.1431296.

32. Um YJ, Choi YJ, Yoo SY. Relationships between smartphone dependency and aggression among middle school students: Mediating and moderating effects of ego-resilience, parenting behaviour, and peer attachment. International Journal of Environmental Research Public Health. 2019;16(19): 3534. DOl:https://doi.org/10.3390/ijerph16193534.
33. Jung YE, Min JA, Shin AY, Han SY, Lee KU, Kim TS, et al. The Korean version of the Connor-Davidson Resilience Scale: An extended validation. Stress Health. 2012;28(4):319-26. DOl:https://doi.org/10.1002/smi.1436.

34. Masten AS. Global perspective on resilience in children and youth. Child Dev. 2014;85(1):6-20. DOl:https://doi.org/10.1111/cdev.12205.

35. Wu G, Feder A, Cohen H, Kim JJ, Calderon S, Charney DS, et al. Understanding resilience. Frontiers in Behavioral Neuroscience. 2013;7:10. DOl:https://doi.org/10.3389/fnbeh.2013.00010.

36. Wisniewski $P$, Jia $H$, Wang $N$, Zheng $S, X u H$, Rosson MB, et al. Resilience mitigates the negative effects of adolescent internet addiction and online risk exposure. Proceedings of the 33rd Annual ACM Conference on Human Factors in Computing Systems. 18-23 April, 2015, Seoul, Republic of Korea. DOl: https://doi.org/10.1145/2702123.2702240.

37. Ekis F. Examination of narcissistic personality traits' predicting level of internet addiction and cyberbullying through path analysis. Educational Science: Theory Practice. 2012;12:1694-706.

38. Li X, Shi M, Wang Z, Shi K, Yang R, Yang C. Resilience as a predictor of internet addiction: The mediation effects of perceived class climate and alienation. 2010IEEE 2nd Symposium on Web Society, 16-17 August 2010, Beijing, China. DOI: https://doi.org/10.1109/SWS.2010.5607478.

39. Zhou P, Zhang C, Liu J, Wang Z. The relationship between resilience and internet addiction: A multiple mediation model through peer relationship and depression. Cyperpsychology Behavior Social Networking. 2017;20(10): 634-9. DOl:https://doi.org/10.1089/cyber.2017.0319.

40. Jung KA, Kim BW. The differences of the ego-resilience and disagreement in parents' rearing attitude to the levels of the smartphone addiction of adolescents. Journal of Digital Convergence. 2015;13(2):299-308. DOl:https:// doi.org/10.14400/JDC.2015.13.2.299.

41. Park SK, Kim JY, Cho CB. Prevalence of internet addiction and correlations with family factors among South Korean adolescents. Adolescence. 2008; 43(172):895-909.

42. Nam CR, Lee DH, Lee JY, Choi AR, Chung SJ, Kim DJ, et al. The role of resilience in Internet addiction among adolescents between sexes. A moderated mediation model. Journal of Clinical Medicine. 2018;7(8):222 DOl:https://doi.org/10.3390/jcm7080222.

43. Chu HS, Tak YR, Lee H. Exploring psychosocial factors that influence smartphone dependency among Korean adolescents. PLOS ONE. 2020;15(5): e0232968-8. DOl:https://doi.org/10.1371/journal.pone.0232968.

44. Kim H. Exercise rehabilitation for smartphone addiction. Journal of Exercise Rehabilitation. 2013;9(6):500-5.

45. Frank R. Approaches to addiction series part 4. Drugs Alcohol Today. 2004;4:30-4.

46. Shonin E, Van Gordon W, Griffiths MD. Mindfulness as a treatment for behavioral addiction. Journal of Addiction Research Therapy. 2014;5(1): 1000e122. DOl:https://doi.org/10.4172/2155-6105.1000e122.

47. Coatsworth JD, Duncan LG, Greenberg MT, Nix RL. Changing parent's mindfulness, child management skills and relationship quality with their youth: Results from a randomized pilot intervention trial. J Child Fam Stud. 2010;19:203-7.

48. Holzel BK, Lazar SW, Gard T, Schuman-Olivier Z, Vago DR, Ott U. How does mindfulness meditation work? Proposing mechanisms of action from a conceptual and neural perspective. Perspectives on Psychological Science. 2011;6:537-59.

49. Tang $Y Y$, Yang L, Leve LD, Harold GT. Improving executive function and its neurobiological mechanisms through a mindfulness-based intervention: Advances within the field of developmental neuroscience. Child Dev Perspect. 2012;6:361-6.

50. Zelazo PD, Lyons KE. The potential benefits of mindfulness training in early childhood: A developmental social cognitive neuroscience perspective. Child Dev Perspect. 2012;6:154-60.

51. Semple RJ, Lee J. Mindfulness-based cognitive therapy for children. In: Baer RA, editor. Mindfulness-based treatment approaches: Clinician's Guide to Evidence Base and Applications. Burlington: Elsevier Academic Press; 2014. pp. 161-85.

52. Lee J, Semple RJ, Rosa D, Miller L. Mindfulness based cognitive therapy for children: Results of a pilot study. Journal of Cognitive Psychotherapy. 2008; 22:15-28.

53. Segal ZV, Williams JG, Teasdale JD. Mindfulness-based cognitive therapy for depression: A new approach to preventing relapse. New York: Guilford Press; 2002.

54. Semple EJ, Lee J, Rosa D, Miller LF. A randomized trial of mindfulness-based cognitive therapy for children: Promoting mindful attention to enhance 
social-emotional resiliency in children. Journal of Children Family Study. 2010;19:218-29.

55. Murray DM, Ronney BL, Hannan PJ, Peterson AV, Ary DV, Biglan A, et al. Intraclass correlation among common measures of adolescents smoking: Estimates, correlates, and application in smoking prevention studies. Am J Epidemiol. 1994;140(11):1038-50.

56. Murray DM, Short BJ. Intraclass correlation among measures related to alcohol use by school aged adolescents: Estimates, correlates and applications in intervention studies. J Drug Educ. 1996;26(3):207-30.

57. Cohen J. A power primer. Psychol Bull. 1992;112(1):155-9.

58. Conner KM, Davidson JR. Development of a new resilience scale: The Conner-Davidson Resilience Scale (CD-RISC). Depression Anxiety. 2003;18(2): 76-82. DOl:https://doi.org/10.1002/da.10113.

59. Yu X, Lau JTF, Mak WWS, Zhang J, Lui WWS, Zhang J. Factor structure and psychometric properties of the Conner-Davidson Resilience Scale among Chinese adolescents. Compr Psychiatry. 2011;52:218-24. DOl:https://doi. org/10.1016/j.comppsych.2010.05.010.

60. Davidson JRT. Connor-Davidson Resilience Scale (CD-RISC) Manual. http:// www.cd-risc.com. Accessed on 3 April 2018.

61. Semple RJ, Lee J. Mindfulness-based cognitive therapy for anxious children: A manual for treating childhood anxiety. Oakland: New Harbinger; 2011.

\section{Publisher's Note}

Springer Nature remains neutral with regard to jurisdictional claims in published maps and institutional affiliations.

Ready to submit your research? Choose BMC and benefit from:

- fast, convenient online submission

- thorough peer review by experienced researchers in your field

- rapid publication on acceptance

- support for research data, including large and complex data types

- gold Open Access which fosters wider collaboration and increased citations

- maximum visibility for your research: over $100 \mathrm{M}$ website views per year

At $\mathrm{BMC}$, research is always in progress.

Learn more biomedcentral.com/submissions 